\title{
Branchial cleft cyst in the parotid gland in a human immunodeficiency virus-negative patient
}

\author{
Yun Yong Park ${ }^{1}$, \\ Jung Soo Yoon ${ }^{1}$, \\ Seong Sik Bang', \\ Hee Chang Ahn ${ }^{1}$ \\ Departments of ${ }^{1}$ Plastic and \\ Reconstructive Surgery and ${ }^{2}$ Pathology, \\ Hanyang University College of \\ Medicine, Seoul, Korea
}

\begin{abstract}
In branchial lymphoepithelial cyst (BLEC), which is also known as branchial cleft cyst, the remnants of a branchial arch develop into a cyst, causing swelling. The first case of BLEC in the parotid gland was reported by Hildebrant in 1895. Since then, BLEC in the parotid gland has continued to be reported, but in rare cases. A 45-year-old man presented to our hospital with a swelling of the left cheek of approximately 6 months' duration. The patient underwent a superficial parotidectomy and was pathologically diagnosed with BLEC. Of note, this was the first case of non-human immunodeficiency virus (HIV)-related BLEC of the parotid gland in South Korea. BLEC is a benign condition, but its treatment depends on the presence of HIV infection. In HIV-negative patients, BLEC does not require a further work-up to evaluate metastasis. Our case report describes the diagnosis and treatment of BLEC in a patient without HIV.
\end{abstract}

Keywords: Branchioma / Parotid gland / Salivary glands

\section{INTRODUCTION}

In branchial lymphoepithelial cyst (BLEC), which also referred to as branchial cleft cyst, the remnant of a branchial arch develops into a cyst, causing swelling [1]. Branchial cleft cysts are classified into four groups depending on which branch cleft they originated from, with locations ranging from the external auditory canal (in first branchial cleft cysts) to the lower part of the neck (in fourth branchial cleft cysts). Branchial cleft cysts in the parotid gland belong to the category of first branchial cleft cysts [2].

Since the first case of BLEC in the parotid gland was reported by Hildebrant in 1895, a total of 70 cases have been reported as of 2018 [1]. The incidence of BLEC in the parotid gland increased with the human immunodeficiency virus (HIV) epidemic, and it now occurs in an estimated 3\% to 6\% of HIV-

\section{Correspondence: Hee Chang Ahn}

Department of Plastic and Reconstructive Surgery, Hanyang University College of

Medicine, 222-1 Wangsimni-ro, Seongdong-gu, Seoul 04763, Korea

E-mail: ahnhc@hanyang.ac.kr

Received January 25, 2019 / Revised March 25, 2019 / Accepted April 19, 2019 positive adults $[3,4]$. However, BLEC of the parotid gland in HIV-negative patients is extremely rare, and the case presented herein is the first such case in South Korea. In this case report, we present the evaluation and treatment of BLEC in the parotid gland in an HIV-negative patient.

\section{CASE REPORT}

A 45-year-old male patient visited the outpatient clinic of the department of plastic surgery with a swelling of the left cheek that had lasted for about 6 months. The patient reported no events that could have caused particular trauma to the left facial area. The size of the patient's cyst had recently been increasing, prompting him to visit a tertiary hospital for examination and treatment (Fig. 1).

Upon physical examination, the swelling of the left cheek was not accompanied by pain and tenderness. There was no change in size or feeling of warmth during eating, and a soft movable mass was palpable. Facial nerve function was intact. We then performed contrast-enhanced facial computed tomography 

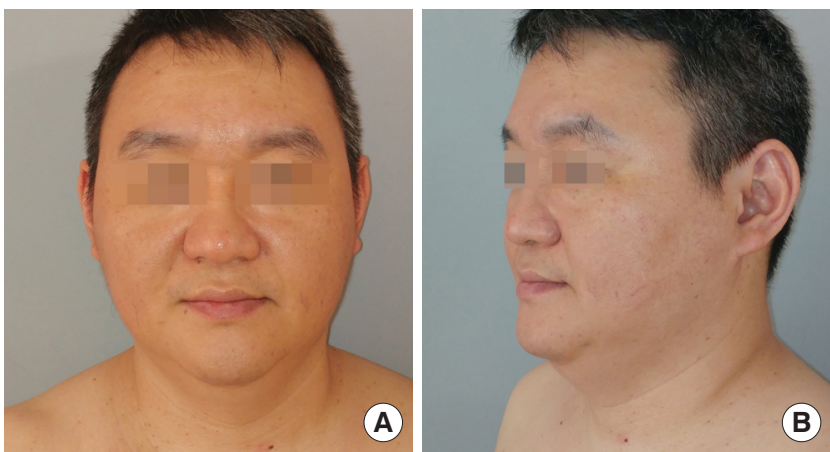

Fig. 1. Preoperative photographs. The patient complained of swelling on the left cheek area. The swelling of the left cheek was not accompanied by pain or tenderness. (A) Frontal view and (B) lateral view.
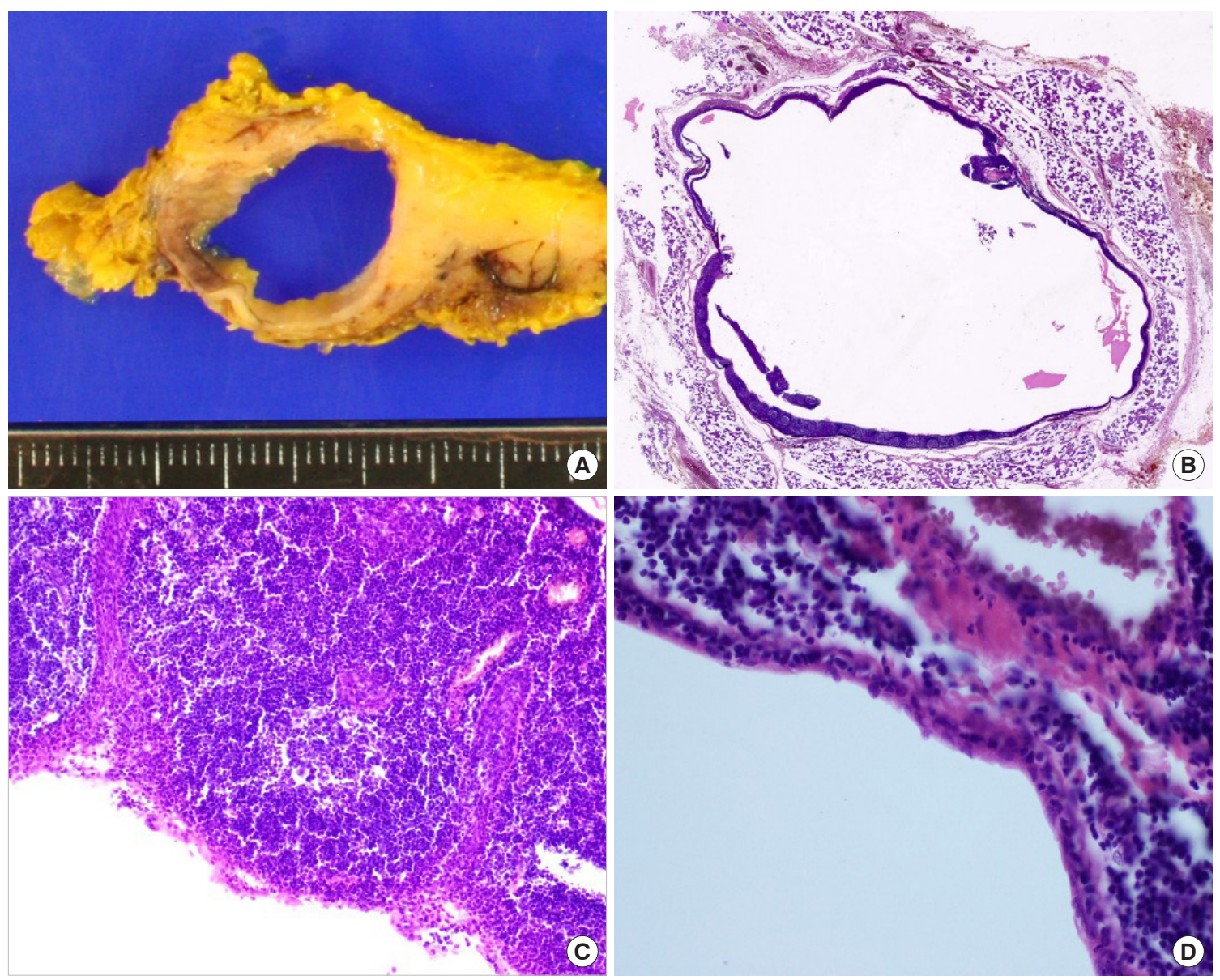

Fig. 3. Histopathologic findings of the lymphoepithelial cyst. (A) The cut surface showed a unilocular cystic lesion, filled with straw-colored fluid (gross findings). (B) On microscopic examination, the dense lymphoid component completely surrounded the cystic cavity (H\&E, scanning view). (C) Lymphoid follicles with a germinal center were identified (H\&E, $\times 200)$. (D) The cyst was lined by attenuated squamous epithelium, and lymphocytic infiltration of the cystic epithelium was prominent (H\&E, $\times 400)$.

(CT) for further evaluation. The differential diagnosis between BLEC and vascular malformations is very important. In general, vascular malformations are hyperdense on contrast-enhanced CT scans, whereas BLECs present as hypodense, thin-

Fig. 2. Preoperative computed tomography. (A, B) The arrow indicates the mass, which was well-defined, located in the superficial lobe, and measured $2.5 \times 1.7 \times 2.1 \mathrm{~cm}$. The findings were suggestive of a benign mass, such as pleomorphic adenoma or sialocele.

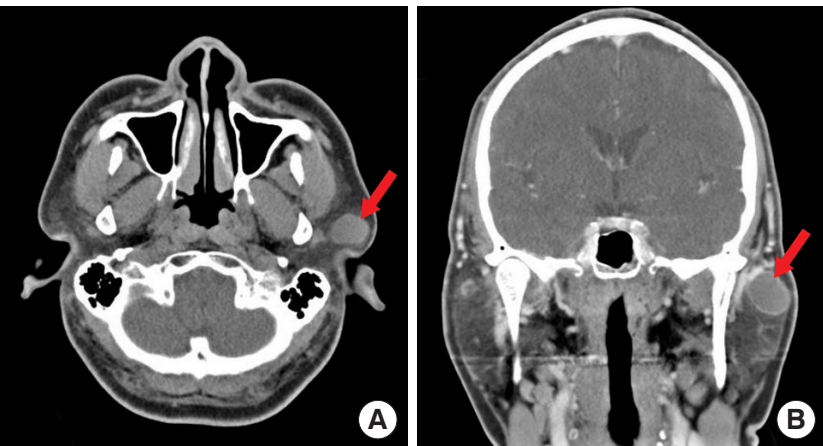

\section{(n)}


sion, such as sialocele or first branchial cleft cyst. The hospital protocol for HIV diagnosis was followed. An HIV rapid test, enzyme-linked immunosorbent assay test, and Western blot were negative. We considered superficial parotidectomy, since the lesion was benign. The patient underwent left superficial parotidectomy under general anesthesia for resection of the mass. During the operation, the soft tissue surrounding the mass showed severe adhesion and fibrosis. After careful dissection and identification of the five main branches of the facial nerve, en bloc mass removal was performed successfully. Sternocleidomastoid (SCM) muscle transfer was performed to prevent Frey syndrome and a depression deformity of the parotid region. The histopathologic report was suggestive of a BLEC of the parotid gland (Fig. 3). Seroma was observed on the 3rd postoperative day and drainage was performed for more than 10 days. Thereafter, the seroma improved and we confirmed the absence of a facial nerve injury. This study was approved by the Institutional Review Board of Hanyang University Seoul Hospital (IRB No. 2019-01-016).

\section{DISCUSSION}

BLEC has been defined as the occurrence of single or multiple cysts within salivary gland lymph nodes $[5,6]$. BLECs are also known as branchial cleft cysts because they develop from the remnant masses of branchial arches. However, the precise pathogenesis of BLEC is controversial, and many theories have been suggested, of which the parotid gland inclusion theory appears to be the most feasible explanation for BLEC formation. According to this proposal, BLECs develop due to the cystic transformation of lymph nodes [1].

BLEC has been associated with HIV infection as part of a diffuse infiltrative lymphocytosis syndrome, in which it is a clinical manifestation of lymphoid hyperplasia in the parotid glands. In recent decades, the HIV epidemic has caused the incidence of parotid gland BLEC to increase [2]. In contrast, BLEC of the parotid gland in HIV-negative patients is extremely rare. In particular, the case described herein is the first case of BLEC of the parotid gland in a HIV-negative patient reported in South Korea.

Histologically, BLEC has a wall lined by stratified epithelium, most commonly squamous, but possibly cuboidal or pseudostratified columnar type. The epithelial component is supported by lymphoid aggregates, demonstrating a well-formed germinal center. Lymphoid hyperplasia is observed inside. The specimens from our patient showed similar pathologic findings (Fig. 3), leading to the pathological diagnosis of BLEC.

BLEC does not recur or metastasize. Therefore, the treatment of BLEC in HIV-negative patients is usually surgical resection. In contrast, HIV-associated BLEC requires observation, repeated aspiration for swelling, and antiretroviral medication. Our patient's mass was confined to the superficial lobe of parotid gland on CT findings, and we therefore performed superficial parotidectomy that included the mass. After superficial parotidectomy, we performed SCM muscle transfer for two purposes. The first was to prevent a depression deformity caused by the mass removal. Second, Frey syndrome can occur after parotidectomy because the parotid gland is not encapsulated [7]. Therefore, in our patient, an SCM muscle flap was used to create a barrier between the parotid gland and overlying skin. After the pathological diagnosis of BLEC, the patient was confirmed to be HIV-negative and the treatment was terminated. There was no complication, and postoperative course was not eventful.

Our case is the first case of BLEC of the parotid gland in South Korea. In addition, it is significant that we successfully treated this patient through our protocol. In conclusion, our case report provides useful information on how to diagnose and treat BLEC in the future.

\section{NOTES}

\section{Conflict of interest}

No potential conflict of interest relevant to this article was reported.

\section{Ethical approval}

The study was approved by the Institutional Review Board of Hanyang University Seoul Hospital (IRB No. 2019-01-016) and performed in accordance with the principles of the Declaration of Helsinki. Written informed consent was obtained.

\section{Patient consent}

The patient provided written informed consent for the publication and the use of his images.

\section{ORCID}

Yun Yong Park https://orcid.org/0000-0003-3594-5891 Jung Soo Yoon https://orcid.org/0000-0003-2462-5702

Seong Sik Bang https://orcid.org/0000-0002-6872-5986

Hee Chang Ahn https://orcid.org/0000-0002-6810-5752

\section{REFERENCES}

1. Joshi J, Shah S, Agarwal D, Khasgiwal A. Benign lymphoepithelial cyst of parotid gland: review and case report. J Oral 
Maxillofac Pathol 2018;22(Suppl 1):S91-7.

2. Krishnamurthy A, Ramshanker V. A type I first branchial cleft cyst masquerading as a parotid tumor. Natl J Maxillofac Surg 2014;5:84-5.

3. Dave SP, Pernas FG, Roy S. The benign lymphoepithelial cyst and a classification system for lymphocytic parotid gland enlargement in the pediatric HIV population. Laryngoscope 2007; 117:106-13.

4. Pillai S, Agarwal AC, Mangalore AB, Ramaswamy B, Shetty S.
Benign lymphoepithelial cyst of the parotid in HIV negative patient. J Clin Diagn Res 2016;10:MD05-6.

5. Varnholt H, Thompson L, Pantanowitz L. Salivary gland lymphoepithelial cysts. Ear Nose Throat J 2007;86:265.

6. Ebrahim S, Singh B, Ramklass SS. HIV-associated salivary gland enlargement: a clinical review. SADJ 2014;69:400-3.

7. Filho WQ, Dedivitis RA, Rapoport A, Guimaraes AV. Sternocleidomastoid muscle flap preventing Frey syndrome following parotidectomy. World J Surg 2004;28:361-4. 